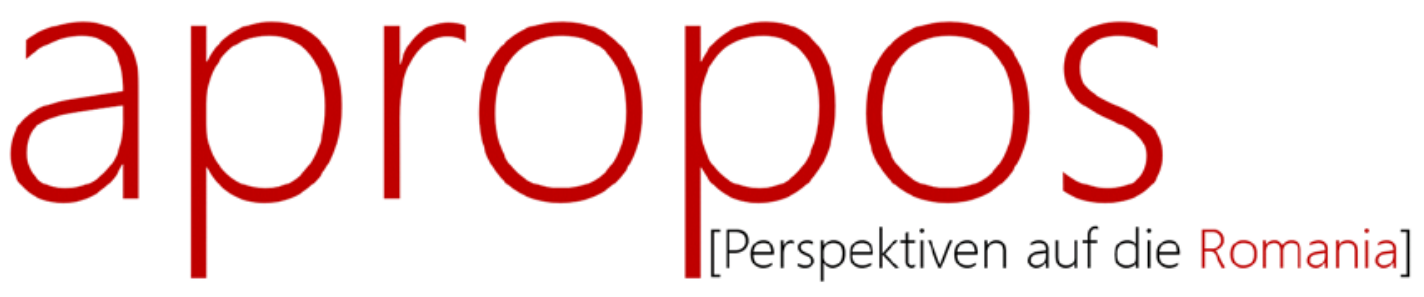

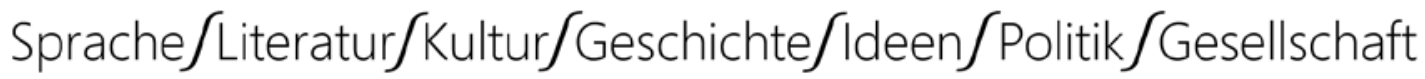

2 | 2019

Rugbykultur (in) der Romania

Rezension von Peraldi, Michel. 2018. Marrakech, ou le souk des possibles. Du moment colonial à l'ère néolibérale. Paris: La Découverte.

Sandra Petermann

apropos [Perspektiven auf die Romania]

hosted by Hamburg University Press

STAATS- UND UNIVERSITÄTS.

BIBLIOTHEK

H A M B UR G

CARL VON OSSIETZKY

2019, 2

pp. $172-176$

ISSN: $2627-3446$

Online

https://journals.sub.uni-hamburg.de/apropos/article/view/1385

Zitierweise

Petermann, Sandra. 2019. „Rezension von Peraldi, Michel. 2018. Marrakech, ou le souk des possibles. Du moment colonial à l'ère néolibérale. Paris: La Découverte.", apropos [Perspektiven auf die Romania] 2, 172-176. doi: 10.15460/apropos.0.1385

Except where otherwise noted, this article is licensed under a Creative Commons Attribution 4.0 International license (CC BY 4.0)

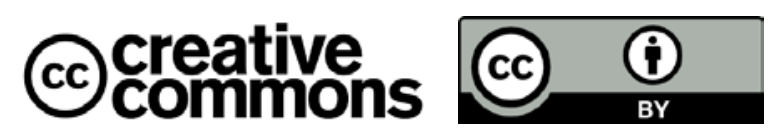


Rezension

\title{
PeRALDI, Michel. 2018. Marrakech, ou le souk des possibles. Du moment colonial à l'ère néolibérale. Paris: La Découverte
}

\author{
Sandra Petermann \\ ist akademische Oberrätin am \\ Geographischen Institut der Johannes \\ Gutenberg-Universität Mainz. \\ s.petermann@uni-mainz.de
}

Keywords

Marrakech - Stadtentwicklung - Stadtsoziologie - Neoliberalisierung - 1912 bis heute

Das neue Werk des französischen Anthropologen Michel Peraldi thematisiert die marokkanische Stadt Marrakech sowohl in historischer als auch aktueller Perspektive als einen Ort vielfältiger Möglichkeiten bzw. vielfältigen Ort des Möglichen. Hierbei fokussiert er auf die Stadtveränderungsprozesse ab 1912 beginnend mit der Protektoratszeit, gefolgt von der postkolonialen Phase bis in die 1990er-Jahre und schließlich die neoliberale Ära ab den 2000er-Jahren - und möchte dadurch nicht nur ein umfassendes Bild von Marrakech der letzten Jahrzehnte liefern, sondern zugleich die Basis einer städtischen Anthropologie formulieren. Peraldi entwickelt hierbei drei sich teils überlappende Zielsetzungen: Erstens möchte er belegen, dass die Stadt Marrakech nicht nur eine Ware ist, die beworben und vermarktet wird, sondern vor allem ein Ort, an dem Menschen auf unterschiedliche Art und Weise leben. Zweitens kritisiert er immer wieder das in seinen Augen bislang wissenschaftlich erzeugte und medial vermittelte Bild von Marrakech und möchte es relativieren bzw. erweitern. In diesem Zusammenhang arbeitet er drittens im Anhang des Buches heraus, dass die Gruppe der in Marrakech neu Zugezogenen sehr heterogen ist und nicht nur aus Vermögenden und Kolonial-Nostalgikern, sondern auch jungen Unternehmern der Mittelklasse besteht, die zum Teil von Prekarität bedroht sind.

Peraldi, der bereits zahlreiche Studien und Publikationen über Migrationsbewegungen im Mittelmeerraum sowie über die Städte Casablanca und Tanger verfasst hat und von 2005 bis 2010 Direktor des Centre Jacques Berque in Rabat war, kennt Marokko seit vielen Jahren. Vor dem Hintergrund dieser Erfahrungen entwirft er nun aus einer soziologisch-anthropologischen Perspektive ein Bild von Marrakech, das durch seinen Wohn-Aufenthalt vor Ort von 2012 bis 2014 sowie 
weitere Reisen nach Marrakech bis 2016 vervollständigt wird. Während dieser Zeit führte er Recherchen durch, sprach mit über 250 Personen und ließ durch eine Schlüsselperson (die er selbst als „marginal man“ (S. 226) charakterisiert) knapp über 100 zugezogene Ausländer quantitativ befragen.

\section{Inhaltliche Zusammenfassung}

Das Buch beginnt am Vorabend der französischen Protektoratszeit in Marrakech mit der Ermordung des französischen Arztes Émile Mauchamp im Jahr 1907, die laut Peraldi die Geburtsstunde des heutigen Marrakechs sowie einer veränderten Idee der französischen Kolonialisierung und kolonialen Beziehungen markiert. Peraldi arbeitet anschließend Phänomene heraus, die für die Stadtentwicklung von Marrakech in der Protektoratszeit entscheidend waren. Zunächst handelt es sich hierbei um die (Stadt)Politik des ersten Generalresidenten Marokkos, Hubert Lyautey, aber auch die Präsenz des Militärs, von französischen Unternehmern und europäischen Reisenden. Peraldi beschreibt die Reisenden der damaligen Zeit als narzisstische Hedonisten, als Eroberer ohne Krieg, als lüsterne Schmatzer und Sammler und interpretiert die französischen Offiziere als zeitgenössische Touristen und Bindeglieder zwischen den ersten Reisenden und heutigen Touristen. Sowoh die touristische Infrastruktur als auch die lokale Bevölkerung von Marrakech blieben von dieser Entwicklung nicht unbeeinflusst, wobei auch der Pascha von Marrakech, Thami El Glaoui, eine zentrale Figur in der kolonialen Transformation der Stadt spielte: in der Medina eröffneten u. a. Hotels, Restaurants und Cafés, in der Ville Nouvelle entstand das später weltbekannte Hotel La Mamounia, ein erster Golfplatz und Casinos wurden gebaut, ,kleine Leute' fanden Beschäftigungsverhältnisse und eine neue städtische Bourgeoisie bildete sich heraus.

In der postkolonialen Phase bis in die 1990er-Jahre, dem zweiten großen Abschnitt des Buches, skizziert Peraldi die Entwicklung Marrakechs von einem eher provinziellen Ort zu einer global beeinflussten Stadt. Zu Beginn dieser Transformation schildert er die überbevölkerte und baufällige Altstadt von Marrakech als einen Ort des Handwerks, einen Ort der Armut und Prekarität, der aber zugleich ab den 1960er-Jahren Destination, Wohnort und Bühne der Hippies, der Beat-Generation und des Jet-Sets war. In den 1980er-Jahren setzte dann ein neuer touristischer Aufschwung ein. Angekurbelt von sowohl neu zugezogenen Ausländern als auch der steigenden touristischen Nachfrage, entwickelte sich unter den Ausländern in Marrakech ein neuer Stil bezüglich Mobiliar und Inneneinrichtung, der traditionelle Techniken modern interpretierte. Die neuen Produkte für den touristischen Markt wurden nur noch zum geringen Teil direkt in den Werkstätten (ateliers) der Produzenten bzw. auf kleinen Märkten (souqs) an ihre Kunden verkauft, sondern zunehmend durch Händler in gewerblich gemieteten Verkaufsläden (bazar) vermarktet. Dies bedeutete einen weiteren Schritt in der ökonomischen und sozialen Transformation der Medina, wobei auch in den 1990er-Jahren zuziehende "entrepreneurs de l'authenticité" (S. 133) und weitere Unternehmer aus der Welt der Mode und des Interior Designs daran arbeiteten, den Grundstein für das spätere ,Logo' Marrakech zu legen.

Im dritten Abschnitt des Buches stellt Peraldi die Stadtentwicklungsprozesse der 
neoliberalen Epoche seit den 2000er-Jahren dar, in der sich Marrakech zu einer multi-nodalen globalisierten Stadt entwickelte. Hierbei beschreibt er ausgewählte Stadtviertel, die architektonische Grammatik von Neubaugebieten, die Privatisierung und Festivalisierung der Stadt sowie einzelne Projekte des Infrastrukturausbaus und Immobiliensektors. Getragen wurden diese Projekte zum einen durch den König selbst, zum anderen durch private und halbstaatliche Unternehmen vornehmlich marokkanischer aber auch internationaler Herkunft. Sie sind bis heute die Akteure des politisch geförderten und sozial durchmischten Wohnungsbaus. Seit 2010 kam es in Marrakech vermehrt zur sozialen Segregation vornehmlich in den Neubauvierteln. Auf der einen Seite wohnen in villes populaires die sozial schwächeren Bevölkerungsgruppen in zunehmend prekären Verhältnissen. Auf der anderen Seite lassen sich statushöhere Bevölkerungsgruppen wie Auslandsmarokkaner sowie Landsleute aus den Städten Casablanca, Rabat, Tanger und Meknès - häufig im Zweitwohnsitz - nieder. Viele der Immobilien stehen in Zeiten der Abwesenheit ihrer Eigentümer leer oder werden temporär vermietet. Für die statushohen Bevölkerungsgruppen entwickelte sich darüber hinaus ein Nischenmarkt für Luxusimmobilien in der Palmaraie und im Ourikatal, für Villen in Gueliz und schließlich für Innenhofhäuser in der Altstadt von Marrakech. Zahlreiche Gästehäuser und Hotels in ausländischer Hand entstanden, Unternehmer wurden vom medialen Bild Marrakechs in Werbung und Printmedien angezogen und neue als showrooms konzipierte Verkaufsräume wurden nicht nur in der Medina, sondern auch im ehemaligen Industrieviertel Sidi Ghanem eingerichtet. Hierdurch fand die lokale männliche und weibliche Bevölkerung einen Zugang zur Arbeitswelt, die allerdings von einer „économie générale diffuse de la domesticité" (S. 214) gekennzeichnet ist.

Im Epilog des Werkes verweist Peraldi trotz aller negativen und fragmentierenden Veränderungen auf positive Initiativen und Ansätze unterschiedlicher Akteure vor Ort, die er als Kritik am primär profitorientierten Kapitalismus und einem zu passiven Staat versteht - wobei die Verantwortlichen und die Bevölkerung vor Ort vor den großen Problemen der Zukunft ihre Augen weitgehend verschließen, wie Peraldi angesichts des rapide sinkenden Grundwasserspiegels eindrücklich veranschaulicht. Im Anhang des Buches stellt Peraldi abschließend die Ergebnisse einer mikrosoziologischen quantitativen Studie aus dem Jahr 2014 vor, in der in Marrakech lebende Europäer von einer Schlüsselperson meist abends an öffentlichen und halböffentlichen Orten befragt wurden. Durch diese nichtrepräsentative Studie möchte Peraldi die Heterogenität der Zugezogenen hinsichtlich Kriterien wie Alter, Beruf, Einkommen, Wohnort, Wohnform, Mobilität und Aufenthaltsstatus in Marokko unterstreichen.

\section{Kritische Würdigung}

Peraldi schafft es entsprechend seiner Zielsetzungen, ein umfassendes Bild der Stadt Marrakech und seiner Bewohner in historischer und gegenwärtiger Perspektive zu zeichnen und spannende Einsichten zu gewähren. Er ermöglicht hierdurch den Leserinnen und Lesern eine erweiterte Perspektive auf Marrakech abseits der medialen Darstellungen in Hochglanzmagazinen und Boulevardpresse. 
Auch bereichert er die wissenschaftliche Diskussion um die aktuellen Stadtveränderungsprozesse auf kommunaler Ebene, wobei er allerdings einige relevante Werke unbeachtet bzw. nicht zitiert zurücklässt. Dies mag man noch verstehen bei deutschsprachigen Publikationen, nicht aber bei französisch- oder englischsprachigen Studien wie zum Beispiel von Kurzac-Souali (2006, 2007, 2011, 2012) oder von Escher und Petermann (2013, 2014). Immer wieder kritisiert Peraldi durchaus zu Recht die Fokussierung auf die Prozesse in der Medina in wissenschaftlichen Publikationen, setzt sich aber inhaltlich nicht mit den Werken auseinander. So bleibt für mich auch seine Kritik an dem in den Werken attestierten Phänomen der Gentrification der Medina nicht nachvollziehbar, die er in Frage stellt, da sie auch in anderen Stadtvierteln außerhalb der Medina (S. 165) sowie nur in wenigen Vierteln innerhalb der Medina zu beobachten sei (S. 186). Wie ein Blick in publiziertes Kartenmaterial klarmacht (Escher \& Petermann 2013) ist das zweite Argument nicht haltbar, da Peraldi das räumliche Ausmaß des Phänomens unterschätzt; die erste Begründung überzeugt durch eine Relativierung auf kommunaler Ebene ebenso wenig. Kritisch zu betrachten ist darüber hinaus, dass Peraldi zwar auf eine beachtlich große Fülle an Gesprächen verweist, kaum jedoch aus ihnen erkennbar zitiert - abgesehen von einer Ausnahme, wo er von Seite 142 bis 147 wörtlich ein Gespräch einfließen lässt. Somit ist eine Nachvollziehbarkeit seiner durchaus spannenden Einsichten nur bedingt gegeben. Dies betrifft auch immer wieder im Text genanntes Zahlenwerk, das beispielsweise auf den Seiten 58, 85 und 182 ohne klar ersichtliche Quellenangaben aufgeführt wird. Wünschenswert wäre auch ein eigenständiges Literaturverzeichnis unabhängig von Endnoten gewesen, das einen schnelleren Überblick über die verwendeten Quellen ermöglicht hätte. Davon unbenommen gelingt es Peraldi, einen sehr guten Überblick über die historische und aktuelle Entwicklung von Marrakech zu zeichnen, dabei relevante Akteure auf lokaler, nationaler und internationaler Ebene herauszuarbeiten sowie die Rolle des Tourismus und Handwerks im Veränderungsprozess klar zu benennen. Auch wird deutlich, dass er einen profunden Einblick in die marokkanische Gesellschaft hat. Das Werk ist anschaulich geschrieben, sodass selbst Nicht-Muttersprachler es gut lesen können.

Kurz: Trotz der genannten Mankos ergänzt das Buch durch seinen Fokus auf die gesamte Stadt und unterschiedliche Akteure sehr gut die wissenschaftliche Debatte um die Stadtveränderungsprozesse und relativiert somit die mediale Darstellung von Marrakech.

\section{Literatur}

Escher, Anton, Sandra Petermann \& Gregor Arnold. 2018. „Das neue Marrakech - eine Materialisierung des Mythos von Tausendundeiner Nacht?" Geographische Rundschau 7/8, 52-57.

EsCHeR, Anton und Sandra Petermann. 2014. "Marrakesh Medina. Neocolonial paradise of lifestyle migrants?" In Contested Spatialities. Lifestyle Migration and Residential Tourism, ed. Michael Janoschka \& Heiko Haas,29-46, Abingdon, New York: Routledge.

EsCher, Anton und Sandra Petermann. 2013. „Facteurs et acteurs de la 
gentrification touristique à Marrakech, Essaouira et Fès." In Médinas immuables? Gentrification et changement dans les villes historiques marocaines (1996-2010), ed. Elsa Coslado, Justin McGuinness \& Catherine Miller, 101-130, Rabat: Centre Jacques-Berque.

Escher, Anton und Sandra Petermann. 2009. Tausendundein Fremder im Paradies? Ausländer in der Medina von Marrakech. Würzburg: ErgonVerlag.

KURZAC-SOUALI, Anne-Claire. 2012. „Représentations et usages renouvelés des médinas gentrifiées du Maroc." In Les quartiers historiques. Pressions, enjeux, action, ed. Etienne Berthold, 51-74, Laval: Presses de I'Université Laval.

KURZAC-SOUALI, Anne-Claire. 2011. „Marrakech, insertion mondiale et dynamiques socio-spatiales locales". Méditerranée-Revue géographique des pays méditerranées 116, 123-132.

KURZAC-SOUALI, Anne-Claire. 2007. „Rumeurs et cohabitation en médina de Marrakech: l'étranger où on ne l'attendait pas." La revue de géopolitique Hérodote 4 (127), 64-88.

KURZAC-SOUALI, Anne-Claire. 2006. Les médinas marocaines: une requalification sélective. Élites, patrimoine et mondialisation au Maroc. Thèse de troisième cycle. Sorbonne. 